\title{
Aging Effect of Starting Solutions for Spherical Silica Synthesis
}

\author{
Naoya ENOMOTO, Akihiro KUMAGAI and Junichi HOJO \\ Department of Applied Chemistry, Faculty of Engineering, Kyushu University, 6-10-1, Hakozaki, Higashi-ku, Fukuoka-shi \\ シリカ球合成の出発溶液のエイジング効果 \\ 榎本尚也·熊谷亮宏 $\cdot$ 北條純一 \\ 九州大学大学院工学研究院応用化学部門, 812-8581 福岡市東区箱崎 6-10-1
}

$812-8581$

\begin{abstract}
Particular attention was paid to the "aging" of starting solution for synthesis of spherical silica by a controlled hydrolysis of alkoxide. Two starting solutions, i.e., an ethanol solution of tetraethylorthosilicate (TEOS) and an ethanol solution of ammonia water, were aged and then mixed to synthesize spheres. Sufficient aging $(\sim$ a week) of the starting solutions resulted in larger sphere size and higher monodispersibility. We infer here that solute molecules are not homogeneously dispersed in the as-dissolved solution even though they appear to be clearly dissolved.

[Received September 9, 2004; Accepted January 20, 2005]
\end{abstract}

Key-words : Silica sphere, Aging, Starting solution, Water-ethanol mixture, Nucleation, Solution structure

\section{Introduction}

When one intends to prepare a starting solution for liquid phase synthesis of ceramic particles or powders, three factors are considered in general. They are species of solvent, species of solute, and their ratio (= concentration). In this work we are going to suggest and notice the fourth factor, the "aging" of starting solution, which means the time after dissolving a solute in a solvent. ${ }^{1)}$

Photonic crystals or colloid crystals are intensively studied recently. ${ }^{2)-4)}$ Crystallinity of photonic crystals is qualified with monodispersibility of particles and the assembly technique of them. It seems that the researchers of photonic crystals purchase monodispersed spheres of silica or polystyrene from companies and focus on the latter technique. If they desire to use spheres with a specific size or composition, it may not be so difficult to synthesize them in their laboratory according to the synthesis methods which were already reported. ${ }^{5)-8)}$ Thus obtained spheres are tolerably uniform at a glance but may not be sufficiently monodispersed when each particle size was examined carefully.

In this paper we focus on the aging effect of the starting solution on the size of silica spheres and its distribution. It will be shown that aging helps to enhance the monodispersibility. Considering that "spheres" in a photonic crystal corresponds to "atoms" in a crystalline substance, a slight improvement of "atomic-level” homogeneity might induce a drastic improvement in the photonics properties.

\section{Experimental}

Commercial reagents of tetraethylorthosilicate (TEOS), ammonia, and absolute ethanol and distilled water were used without further purification. Concentration was fixed at TEOS $=0.1 \mathrm{~mol} / \mathrm{dm}^{3}, \quad \mathrm{NH}_{3}=1.8 \mathrm{~mol} / \mathrm{dm}^{3}, \quad \mathrm{H}_{2} \mathrm{O}=7.6 \mathrm{~mol} /$ $\mathrm{dm}^{3}$ in ethanol solvent as a whole. Half of total ethanol is used to prepare an ethanolic solution of TEOS (denoted as Solution A). And the rest half of ethanol is used to prepare an ethanolic solution of ammonia water (denoted as Solution B). Derivative experimental methods will be mentioned in later sections.

Starting solutions were prepared by mixing the reagents for a while, and then tightly sealed in a brown glass container and quiescently aged at room temperature in the shade. Synthesis of spheres was executed by mixing the two solutions in a transparent beaker at a constant temperature of $10^{\circ} \mathrm{C}$ using a magnetic stirrer. Incubation period (IP) of the precipitation was estimated by carefully inspecting a development of turbidity in the reacting solution by naked eyes under a suitable lighting. This rough method gave sufficiently reproducible and meaningful results. The longest reaction time adopted was 120 min. For the purpose of investigating the growth of spheres, a small amount of the reacting solution were occasionally sampled and diluted in ethanol to stop the reaction and were subjected to scanning electron microscopy (SEM). Final product was centrifuged, washed several times by ethanol, and dried at $60^{\circ} \mathrm{C}$ in an oven.

Size of spheres was measured from SEM pictures. Several hundreds of spheres were counted and ranked in order of size. Sphere size at the top 10\% (D10) and that at the bottom 10\% (D90) were used to evaluate the monodispersibility (D90/ D10) of each sample. The closer the value of D90/D10 approaches to unity, the higher the monodispersibility is.

\section{Results and discussion}

3.1 Effect of aging on formation and size of spheres Figure 1 (a) shows that the incubation period (IP) for the appearance of turbidity becomes longer as aged. Growth curves in Fig. 2 indicate that the reaction time of $120 \mathrm{~min}$ is enough. The shape of the growth curves for fresh ( 0 day) and aged (3, 7 days) runs are similar but the final size is larger when the aged solutions were used. Monodispersibility and median size of the spheres after 120 min's synthesis are shown in Figs. 1(b) and (c), respectively. It is obvious that aging process enhanced the both.

Here we calculate the number of nucleus with a simple assumption. At first, how much mole of $\mathrm{SiO}_{2}(\mathrm{M} . \mathrm{W} .=60 \mathrm{~g}$ / mol) are involved in a single sphere (density; $\rho \approx 1.8 \mathrm{Mg} / \mathrm{m}^{3}$ ) with a diameter of $D[m]$ ? This may represent the degree of polymerization $\left(\mathrm{SiO}_{2}\right)_{n}$;

$$
n[\mathrm{~mol} / \text { particle }]=\frac{\frac{4}{3} \pi\left(\frac{D}{2}\right)^{3} \cdot \rho}{M . W .}
$$

Assuming that the $C\left(=0.1 \mathrm{~mol} / \mathrm{dm}^{3}\right)$ TEOS is fully hydrolyzed to form monodispersed spheres of $D[m]$, the solution 


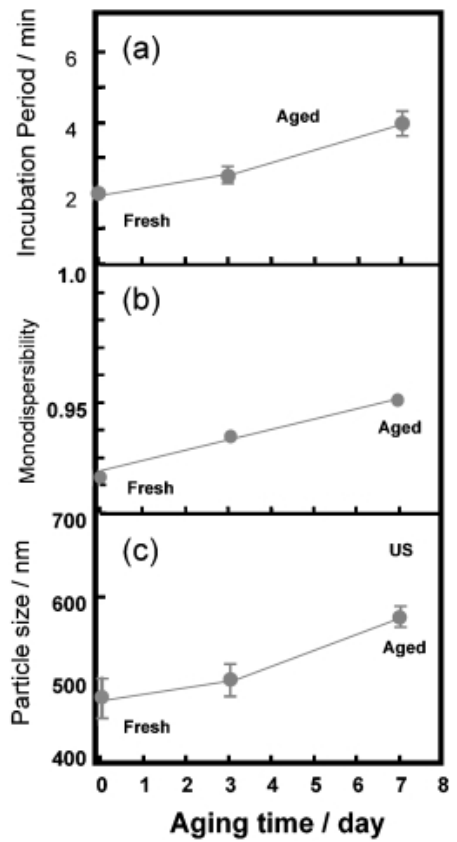

Fig. 1. Effect of aging on (a) incubation period, (b) monodispersibility, and (c) particles size of silica sphere. Reaction time was 120 $\min$ for $(b)$ and $(c)$.

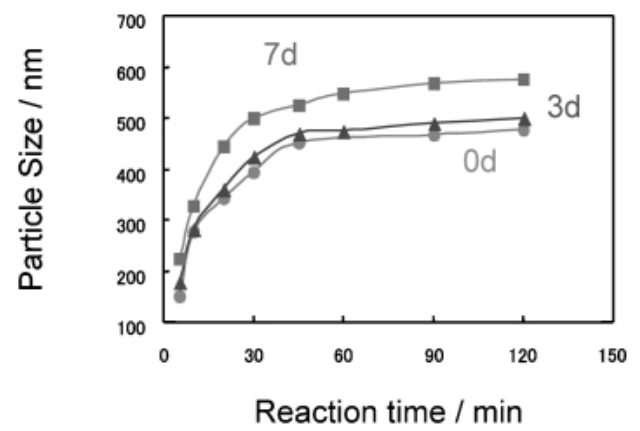

Fig. 2. Growth curves of silica spheres synthesized from fresh and aged solutions. Aging time was 3 or 7 days.

should contain the following number of particles per unit volume.

$$
\frac{C}{n}\left[\text { particles } / \mathrm{dm}^{3}\right]=\frac{6 C \cdot M \cdot W \cdot}{\pi \rho} D^{-3}
$$

This value can represent the number of nucleus $(N)$. Comparing the size of spheres prepared from fresh solutions $\left(D_{\text {fresh }}\right.$ $=470 \mathrm{~nm})$ and that from 7days-aged solutions $\left(D_{7 \mathrm{~d}}=580\right.$ $\mathrm{nm})$, the number ratio of nucleus $\left(N_{7 \mathrm{~d}} / N_{\text {fresh }}\right)$ equals to $\left(D_{\text {fresh }} / D_{7 \mathrm{~d}}\right)^{3}$, which is calculated to be about 0.53 . In conclusion, the number of nucleus decreased to half when the starting solutions were aged for a week. It should be related to the fact that we could catch the clear difference in IP's for fresh and aged runs in Fig. 1 (a).

Needless to say, it is quite easy to prepare a "homogeneous" starting solution because either TEOS or water is very soluble with ethanol. However, we must notice here that a "transparent" solution means that it is only scattering-free from visible lights. Considering the size of their wavelength, "transparent homogeneity" does not at all guarantee the homogeneity at

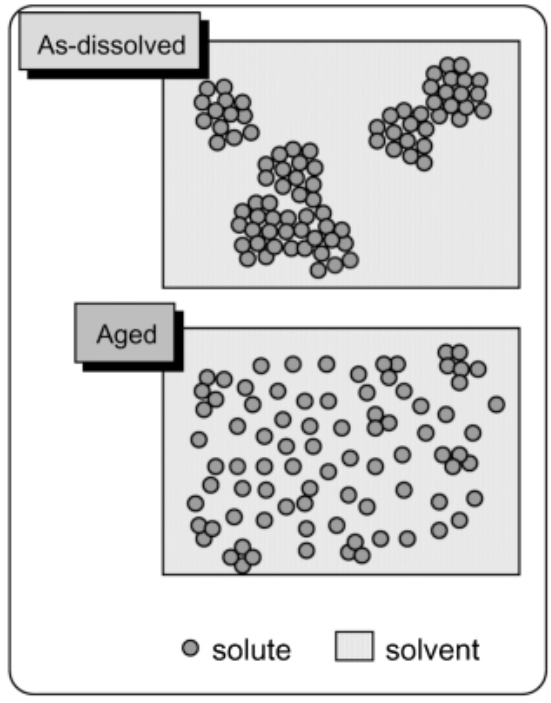

Fig. 3. Assumed schematic for the change of solution structure during aging. Large clusters in the as-dissolved solution may induce heterogeneous nucleation.

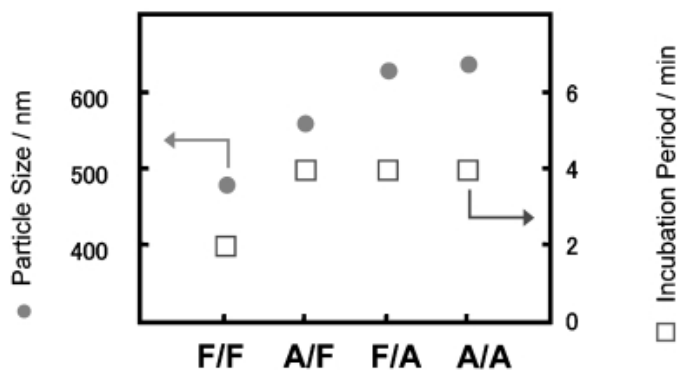

Mixing index $; A / B=($ Fresh or $\underline{\text { Aged }}) /($ Fresh or $\underline{\text { Aged }})$

Fig. 4. Results of crossover experiment. Notation "F/A" means that Fresh Solution A and Aged Solution B were mixed to react. Reaction time was $120 \mathrm{~min}$.

molecular level. One hypothesis to explain the results in Fig.1 is shown in Fig. 3. Just after a solute is dissolved in a solvent, it may not be well dispersed at microscopic level. Large clusters in the as-dissolved (fresh) solution act as a heterogeneous nucleation site to degrade monodispersibility. After they vanished by aging, nucleation would be more homogeneous and particle size becomes larger.

\subsection{Crossover experiment}

In the above experiments, two solutions of $\mathrm{A}$ and $\mathrm{B}$ were equally aged and then reacted. In this section, fresh solution of $\mathrm{A}$ and aged solution of $\mathrm{B}$, and vice versa, were compared. Notation "F/A" means the reaction between Fresh Solution A and the Aged Solution B. Aging time was 7 days. Figure 4 shows the final sphere size and the IP for four different combinations. The IP values are found to be almost the same when either solution was aged. Comparing the sphere size of $\mathrm{A} / \mathrm{F}$ and $\mathrm{F} / \mathrm{A}$, the latter is found to be larger. This means that the aging of Solution B is a little more efficient than that of Solution A. According to the hypothesis that a well-mixed solution yields larger spheres, as described in the former section, it is inferred that the "microscopic" miscibility of water-ethanol mixture is less than that of TEOS-ethanol mixture.

It is well-known that water and ethanol are miscible through 


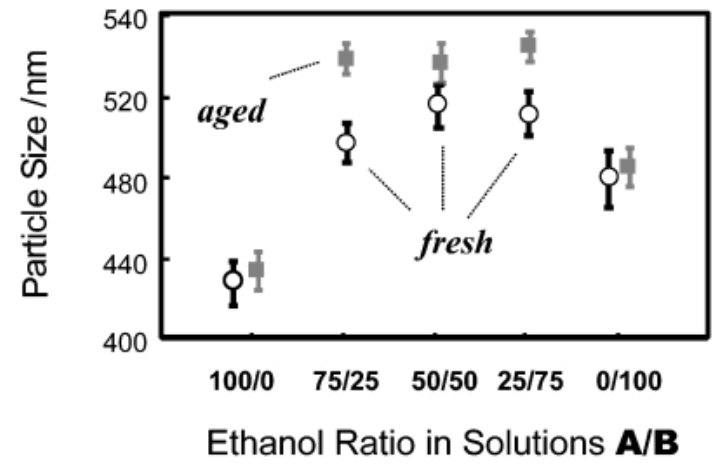

Fig. 5. Effect of ethanol distribution. Notation " $25 / 75$ " indicates $25 \%$ of total ethanol was used for Solution A, and the rest $75 \%$ was used for Solution B. Note that total amount of ethanol is always constant. Aging time was 7 days. Reaction time was $120 \mathrm{~min}$. Aged and fresh are indicated by $\square$ and $\bigcirc$, respectively.

a whole composition. Here we would like to propose a simple question -.-Is it truly easy to mix ethanol and water? It should be noticed here that alcohol $\left(\mathrm{C}_{n} \mathrm{H}_{2 n+1} \mathrm{OH}\right)$ with larger alkyl group $(n>\sim 5)$ is immiscible with water like oil. Then, we may reasonably say that ethanol $(n=2)$ is less miscible with water than methanol $(n=1)$. It is reported from a medical point of view that the intoxication of a water-ethanol mixture or liquor is decreased by aging. ${ }^{9), 10)}$ In short, experimental mice dosed with well-aged mixture recover from hangover earlier than those dosed with less-aged one. They also detected curious and obvious changes due to aging. Thermal analysis ${ }^{11)}$ indicated that a freezing point tends to be lower for the wellaged mixture. ${ }^{2} \mathrm{H}-\mathrm{NMR}$ spectroscopy ${ }^{9}$ showed that relaxation time $\left(T_{1}\right)$ of the well-aged mixture is slightly but essentially longer than that of less-aged one. According to their interpretation, aging makes the solution structure more compact and homogeneous. This can also be applicable to explain our results in Fig. 1.

\subsection{Effect of ethanol distribution on sphere size}

In the above experiments, solvent ethanol were equally distributed to Solutions A and B. In this series, it was distributed at different mass $\%$ for them. Notation " $25 / 75$ " indicates $25 \%$ of total ethanol was used to dissolve TEOS (Solution A), while the rest $75 \%$ was used to dissolve ammonia water (Solution B). Total amount of ethanol is always constant. Figure 5 shows the 7days-aging effect for different distribution of ethanol solvent. It should be noted that aging effect is obvious when the both solutions contain some amount of ethanol (75/ 25 to $25 / 75)$. While, there is rare effect of aging as for $100 / 0$ and $0 / 100$. Comparing these two, the latter gave a larger sphere size. It means that dissolution of water is more effective to obtain larger spheres than that of TEOS. This is the same tendency as the results of the crossover experiment in Fig. 4.

3.4 Effect of intentional addition of a little water

It should be additionally mentioned that we briefly examined the effect of moisture in ethanol because it may be involved intrinsically or absorbed from atmosphere during the process. Although we employed the absolute ethanol and always used a new bottle of it to start a series of experiment, a trace of water in ethanol may be inevitable in practice. In order to look at the effect of water, 1 mass $\%$ of water was previously added to the Solution A prior to aging. As a result, the difference of the sphere size for the runs with and without water addition was not apparent. It is inferred that the hydrolysis of TEOS in the absence of (ammonia) catalysis at low (room) temperature is so slow that a trace of water in Solution $\mathrm{A}$ is negligible in the present aging condition.

Results in the previous sections (Figs. 4 and 5) show that the aging of Solution B appears to be more influential, where chemical reaction among ammonia, water, and ethanol is scarcely present. Accordingly, we consider that the essence of aging in this work is not chemical but physical as shown in Fig. 3. This is a similar understanding as in Refs. 9-11.

3.5 Aging effect of solution for growth of seed spheres

When one desires to prepare larger particles, it is a convenient way to grow seed particles in a suitable condition. In this section, once prepared spheres were grown in fresh or aged solution. Seed spheres of $\sim 500 \mathrm{~nm}$ were prepared as usual at TEOS: $\mathrm{NH}_{3}: \mathrm{H}_{2} \mathrm{O}=0.1: 1.8: 7.6 \mathrm{~mol} / \mathrm{dm}^{3}$, and were washed and dried. Solutions $\mathrm{A}$ and $\mathrm{B}$ with the equal distribution of ethanol were equally aged for $0-14$ days. The $1 / 8$ portion of the dried powder was processed for 120 min by pouring the same amount of Solutions A and B as the seeds were prepared. If the newly supplied component is wholly deposited on each seed, the spheres should grow to twice as large $\left[(1 / 8)^{-1 / 3}\right.$ $=2]$.

Figures $6(a)-(c)$ show micrographs of spheres grown by fresh and aged solutions. Size of spheres was no more monodispersed but has a bimodal (or more) distribution. This means that an extra nucleation occurred during the growth. When we note the number of approximately doubly grown spheres, it is less than half for the growth with the fresh solution. However, the number is obviously increasing with increasing aging time (Fig. 6(d)). Although 14 days are not enough for a perfect monodispersed growth, it has been proved that aging has a definite power to reduce the extra nucleation to degrade the monodispersibility.

\section{Conclusion}

In the wet-chemical synthesis of particles and powders using precipitation phenomena, we may not pay much attention to starting solutions if only they are visibly transparent and clear. Sometimes we use a stock solution for a series of experiment, which could take more than 1 week. It was found in this work that aging of starting solutions for silica spheres synthesis delayed the incubation period, increased the sphere size, and enhanced the monodispersibility. It was approximately estimated that the number of nucleus decreased to $\sim 50 \%$ by aging at room temperature for a week. Moreover, aging is also useful to decrease an extra nucleation when seed spheres are grown to larger size.

We are presuming that this is due to the microscopic homogenization in solution structure. This sort of thing may likely occur with an organic solvent but may unlikely occur in the aqueous system since water strongly hydrates to solute ions to disperse them effectively due to the high value of dielectric constant $(\varepsilon=89.5)$. The fourth factor "aging" of the starting solution could be a useful parameter to control the nucleation and growth of them.

\section{References}

1) Enomoto, N., Shiihara, J., Hongo, T. and Nakagawa, Z., $J$. Ceram. Soc. Japan, Vol. 107, pp. 285-288 (1999).

2) Tang, F., Fudouzi, H., Zhang, J. and Sakka, Y., Scripta. Mater., Vol. 49, pp. 735-740 (2003).

3) Fudouzi, H. and Xia, Y. N., Adv. Mater., Vol. 15, pp. 892-896 (2003).

4) Okubo, T., Prog. Colloid Polym. Sci., Vol. 124, pp. 112-115 (2003).

5) Ogihara, T., Nakajima, H., Yanagawa, T., Ogata, N., Yoshida, K. and Matsushita, N., J. Am. Ceram. Soc., Vol. 74, pp. 

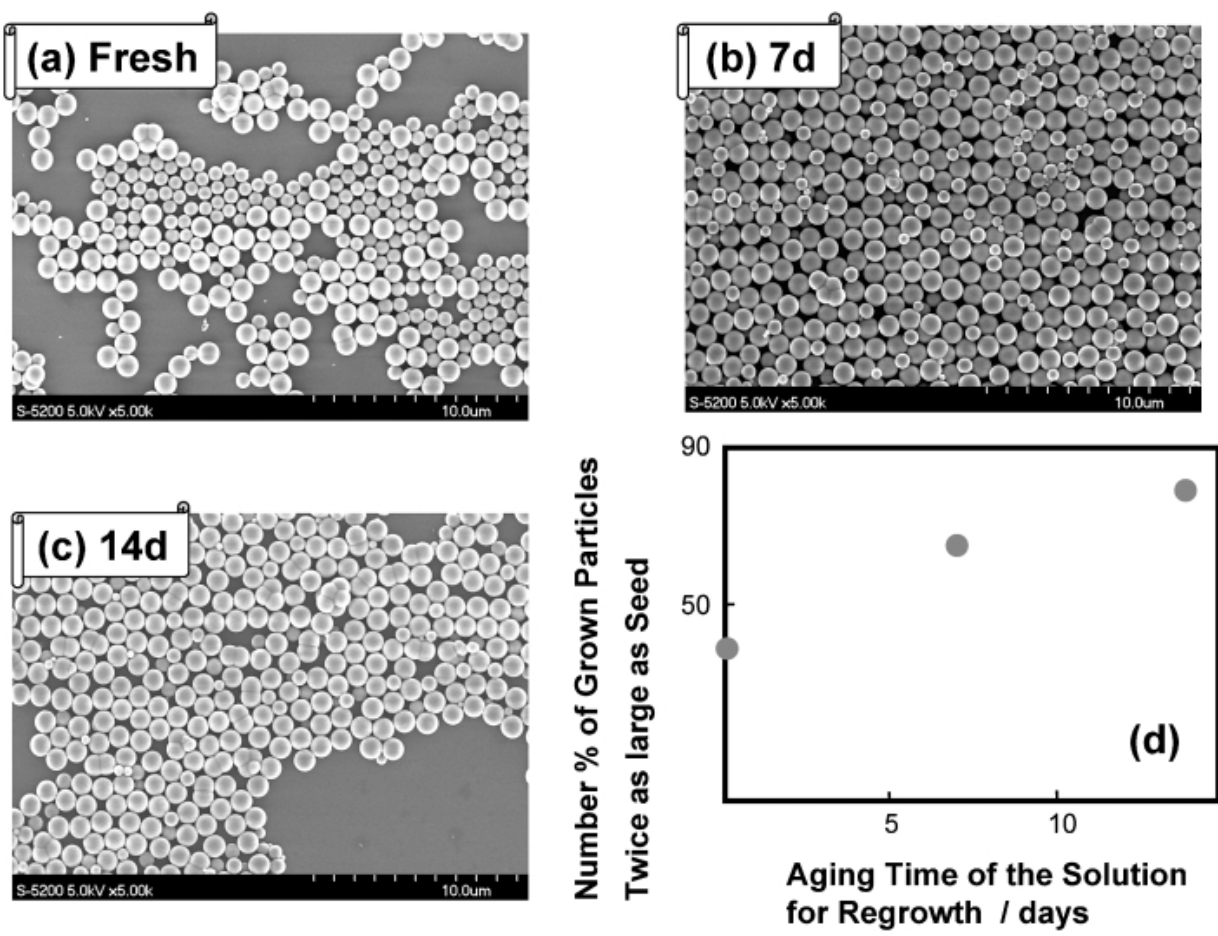

Fig. 6. Aging effect in growth of seed spheres; SEM photographs of spheres grown by (a) fresh solutions, (b) 7 days-aged solutions, and (c) 14 days-aged solutions. The number of approximately doubly grown spheres were counted and plotted in the graph (d).

2263-2269 (1991).

6) Nakanishi, K., Takamiya, Y. and Shimodaira, T., J. Ceram. Soc. Japan, Vol. 94, pp. 1023-1028 (1986).

7) Ikemoto, T., Mizutani, N., Kato, M. and Mitarai, Y., J. Ceram. Soc. Japan, Vol. 93, pp. 585-586 (1985).

8) Barringer, E. A. and Bowen, H. K., J. Am. Ceram. Soc., Vol. 65, C199-C201 (1982).

9) Matsushita, K., Nishina, M., Asakura, T., Kamei, S., Suzuki, M. and Yabe, K., Physiol. Chem. Phys. \& Med. NMR, Vol. 32, pp. 13-19 (2000).

10) Haseba, T., Matsushita, K., Asakura, T., Kameyama, K., Tamaki, T., Okouchi, S., Watanabe, T. and Uedaira, H., Alcoholism: Clinical and Experimental Res., Vol. 17, pp. 963-967 (1993).

11) Haseba, T., Mikami, K., Ohno, Y., Kameyama, K., Tsujii, T., Saeki, C., Asakura, T. and Uedaira, H., "Proc. 14th Mtg. Int'l Assoc.Forensic Sci., Vol. 2,” Shunderson Comm., Canada (1997) pp. 322-324. 